\title{
Comportamento da Mecânica Pulmonar após a Aplicação de Protocolo de Fisioterapia Respiratória e Aspiração Traqueal em Pacientes com Ventilação Mecânica Invasiva*
}

\author{
Behavior of the Lung Mechanics after the Application of \\ Protocol of Chest Physiotherapy and Aspiration Tracheal \\ in Patients with Invasive Mechanical Ventilation
}

Fernanda Kusiak da Rosa', Cláudia Adegas Roese ${ }^{2 \dagger}$, Augusto Savi ${ }^{3}$, Alexandre Simões Dias ${ }^{4 t}$, Mariane Borba Monteiro ${ }^{5 t}$

\section{RESUMO}

JUSTIFICATIVA E OBJETIVOS: A fisioterapia respiratória (FR) em pacientes submetidos a suporte ventilatório invasivo, atua diretamente no sistema ventilatório podendo alterar a mecânica pulmonar através da complacência pulmonar dinâmica (Cdyn) e da resistência do sistema respiratório (Rsr). Porém, as alterações descritas após a realização de FR permanecem controversas. O objetivo deste estudo foi avaliar as alterações da mecânica pulmonar em pacientes em ventilação mecânica invasiva (VMI).

1. Fisioterapeuta Graduada pelo Centro Universitário Metodista IPA. 2. Acadêmica de Fisioterapia do Centro Universitário Metodista IPA. 3. Fisioterapeuta do Centro de Tratamento Intensivo Adulto do Hospital Moinhos de Vento.

4. Fisioterapeuta. Mestre em fisiologia pela Universidade Federal do Rio Grande do Sul (UFRGS); Doutor em fisiologia pela UFRGS; Coordenador do Mestrado em Reabilitação e Inclusão do Centro Universitário Metodista IPA.

5. Fisioterapeuta. Especialista em Fisiologia do Exercício pela UFRGS; Mestre em Ciências Médicas pela UFRGS; Docente do Centro Universitário Metodista IPA.

†Grupo de Pesquisa em Distúrbios Respiratórios e Reabilitação do Centro Universitário Metodista IPA.

${ }^{*}$ Recebido do Centro Universitário Metodista IPA de Porto Alegre, Porto Alegre, RS

Apresentado em 24 de janeiro de 2007

Aceito para publicação em 12 de abril de 2007

Endereço para correspondência:

Cláudia Adegas Roese

Av. General Barreto Viana, $n^{\circ} 361 / 201$

91330-630, Porto Alegre, RS

Fones: (51) 3338-1349 - 8438-3424

E-mail: cladegas@yahoo.com.br

(C)Associação de Medicina Intensiva Brasileira, 2007
MÉTODO: Foi realizado estudo prospectivo e aleatório, controlado do tipo cruzado, incluindo pacientes com mais de 48 horas em VMI. Os protocolos de fisioterapia respiratória (PF) e de aspiração traqueal isolada (PA) foram aleatorizados para a ordem de aplicação, com intervalo de 24 horas entre eles. Dados da mecânica pulmonar e das variáveis cardiorrespiratórias foram coletados antes da aplicação do protocolo, imediatamente após; 30 minutos e 120 minutos após a aplicação dos protocolos.

RESULTADOS: Doze pacientes completaram o estudo. A pneumonia foi a causa mais comum de insuficiência respiratória (IRpA). Não houve diferença estatisticamente significativa entre os grupos em relação à Cdyn, volume de ar corrente (VAC) e volume-minuto (VM). A Rsr diminuiu de forma significativa imediatamente após (de $10,4 \pm 3 \mathrm{cmH}_{2} \mathrm{O} / \mathrm{L} / \mathrm{seg}$ para $8,9 \pm 2 \mathrm{cmH}_{2} \mathrm{O} / \mathrm{L} / \mathrm{seg} ; \mathrm{p}<$ 0,02), 30 minutos após (de 10,4 $\pm 3 \mathrm{cmH}_{2} \mathrm{O} / \mathrm{L} /$ seg para 9 $\pm 2 \mathrm{cmH}_{2} \mathrm{O} / \mathrm{L} / \mathrm{seg} ; \mathrm{p}<0,01$ ) e $120 \mathrm{~min}$ após (de 10,4 \pm 3 $\mathrm{cmH}_{2} \mathrm{O} / \mathrm{L} /$ seg para $9 \pm 2 \mathrm{cmH}_{2} \mathrm{O} / \mathrm{L} /$ seg; $\mathrm{p}<0,03$ ) a aplicação do protocolo de fisioterapia respiratória. Quando comparado com o protocolo de aspiração traqueal isolada foi significativamente menor nos momentos $30(9 \pm$ $2 \mathrm{cmH}_{2} \mathrm{O} / \mathrm{L} / \mathrm{seg}$ versus $\left.10,2 \pm 2 \mathrm{cmH}_{2} \mathrm{O} / \mathrm{L} / \mathrm{seg} ; \mathrm{p}<0,04\right)$ e 120 minutos $\left(9 \pm 2 \mathrm{cmH}_{2} \mathrm{O} / \mathrm{L} /\right.$ segundo versus $10,4 \pm 3$ $\mathrm{CmH}_{2} \mathrm{O} / \mathrm{L} /$ seg; $\left.\mathrm{p}<0,04\right)$.

CONCLUSÕES: O protocolo de fisioterapia respiratória foi eficaz na diminuição da Rsr quando comparado com o protocolo de aspiração. Essa diminuição manteve-se duas horas após a sua aplicação, o que não ocorreu quando realizada apenas a aspiração traqueal isolada.

Unitermos: aspiração traqueal, desobstrução de via aérea, fisioterapia respiratória, mecânica pulmonar, ventilação mecânica. 


\section{SUMMARY}

BACKGROUND AND OBJECTIVES: The chest physiotherapy $(\mathrm{CP})$ in patients submitted to invasive support ventilation acts directly in the breathing system, and it could alter the lung mechanics through the dynamic lung compliance (DynC) and resistance of the breathing system (Rbs). However the alterations after the accomplishment of $\mathrm{CP}$ are still controversy. The objective of this study was to evaluate the alterations of the lung mechanics in patients in invasive mechanical ventilation (IMV).

METHODS: It was a prospective, randomized, and controlled and crossover study, with patient with more than 48 hours in IMV. The protocol of chest physiotherapy and isolated tracheal aspiration they were randomized for the application order with a window of 24 hours among them. Data of lung mechanics and its varied cardiorespiratory were collected moments before the protocol, immediately after the application of the protocol, 30 minutes and 120 minutes after the application of the protocols.

RESULTS: Twelve patients completed the study. Pneumonia was the mean cause respiratory failure (RF). There was not statistical difference among the groups in relation to Cdyn, volume tidal (Vt) and volume minute (Ve). Rbs decreased in a significant way immediately after (of $10.4 \pm 3 \mathrm{cmH}_{2} \mathrm{O} / \mathrm{L} / \mathrm{seg}$ for $8.9 \pm 2 \mathrm{cmH}_{2} \mathrm{O} / \mathrm{L} / \mathrm{seg}$; $\mathrm{p}<0.02$ ), 30 minutes after (of $10.4 \pm 3 \mathrm{cmH}_{2} \mathrm{O} / \mathrm{L} / \mathrm{seg}$ for $\left.9 \pm 2 \mathrm{cmH}_{2} \mathrm{O} / \mathrm{L} / \mathrm{seg} ; \mathrm{p}<0.01\right)$ and 120 minutes after (of $10.4 \pm 3 \mathrm{cmH}_{2} \mathrm{O} / \mathrm{L} /$ seg for $9 \pm 2 \mathrm{cmH}_{2} \mathrm{O} / \mathrm{L} /$ seg; $\mathrm{p}<$ 0.03) application the protocol of chest physiotherapy. When compared with the protocol of isolated tracheal aspiration it was significantly smaller in the $30(9 \pm 2$ $\mathrm{cmH}_{2} \mathrm{O} / \mathrm{L} /$ seg versus10.2 $\pm 2 \mathrm{cmH}_{2} \mathrm{O} / \mathrm{L} / \mathrm{seg} ; \mathrm{p}<0.04$ ) and 120 minutes $\left(9 \pm 2 \mathrm{cmH}_{2} \mathrm{O} / \mathrm{L} / \mathrm{seg}\right.$ versus $10.4 \pm 3$ $\left.\mathrm{cmH}_{2} \mathrm{O} / \mathrm{L} / \mathrm{seg} ; \mathrm{p}<0.04\right)$.

CONCLUSIONS: The protocol of chest physiotherapy was effective in the decrease of Rsr when compared with the aspiration protocol. That decrease was maintained for two hours after its application, what did not happen when only the just accomplished the tracheal aspiration was performed isolated.

Key Words: airway clearance, chest physiotherapy, lung mechanics, mechanical ventilation, tracheal aspiration.

\section{INTRODUÇÃO}

Em muitos casos de insuficiência respiratória aguda (IRpA), o manuseio imediato ocorre através da in- tubação traqueal e do uso da ventilação mecânica invasiva (VMI) como suporte de vida nos pacientes com sofrimento ventilatório'.

Apesar de a VMI ser uma alternativa comumente empregada nos Centros de Tratamento Intensivo (CTI), sua utilização predispõe riscos aos pacientes. As complicações relacionadas ao seu uso incluem lesão traqueal, barotrauma e/ou volutrauma, diminuição do débito cardíaco e toxicidade pelo uso do oxigênio². Além disso, pacientes em VMI tendem a acumular secreções respiratórias devido à tosse ineficaz, em detrimento do não fechamento da glote e prejuízo no transporte do muco pela presença do tubo traqueal. A retenção de secreção contribui para episódios de hipoxemia, atelectasia e pneumonia associada ao ventilador ${ }^{3}$.

As técnicas de fisioterapia respiratória (FR), objetivando o aumento da permeabilidade das vias aéreas e prevenção do acúmulo de secreções brônquicas, são amplamente utilizadas nos CTI. As técnicas desobstrutivas incluem a compressão torácica manual (CTM), hiper-insuflação manual (HM), drenagem postural (DP), aspiração traqueal, dentre outras ${ }^{4}$.

Acredita-se que a higiene brônquica proporciona melhora na mecânica respiratória através do aumento da complacência pulmonar dinâmica (Cdyn) e diminuição da resistência do sistema respiratório $(\mathrm{Rsr})^{5,6}$. Hodgson e col. avaliaram a técnica de HM em 18 pacientes com VMI comparando com aspiração traqueal isolada. A Cdyn aumentou em 30\% após a técnica de HM e maior volume de secreção pulmonar foi removido após a sua realização ${ }^{7}$. Em contrapartida, Unoki e col. não encontraram diferença estatisticamente significativa ao comparar técnicas manuais aplicadas sobre o tórax com a aspiração traqueal isolada em pacientes que estavam em $\mathrm{VMI}^{8}$.

Apesar da maioria dos estudos compararem as técnicas isoladamente, a combinação entre elas é a prática mais utilizada por fisioterapeutas que atuam nos CTI com o objetivo de remover secreções respiratórias. As pesquisas apresentam protocolos variados, com tempo e aplicação de técnicas distintas acarretando em resultados contraditórios com relação à mecânica pulmonar.

O objetivo deste estudo foi analisar as alterações da mecânica pulmonar e dos parâmetros cardiorrespiratórios relacionados a um protocolo fisioterapêutico em pacientes com VMI comparando-o com um protocolo de aspiração traqueal. 


\section{MÉTODO}

Foi realizado estudo prospectivo e aleatório, controlado do tipo cruzado, com amostra não probabilística intencional, desenvolvido de setembro a novembro de 2005, no Centro de Tratamento Intensivo do Hospital Moinhos de Vento em Porto Alegre. A pesquisa foi aprovada pelo Comitê de Ética em Pesquisa do Centro Universitário Metodista IPA e do referido hospital. Os familiares ou responsáveis dos pacientes assinaram um Termo de Consentimento Livre e Esclarecido.

Foram incluídos no estudo os pacientes com diagnóstico de IRpA, submetidos à VMI por período superior a 48 horas através do respirador EVITA II e IV (Dräger; Alemanha). Os critérios de exclusão foram a presença de sinais clínicos de instabilidade hemodinâmica (pressão arterial média $<60 \mathrm{mmHg}$ ), broncoespasmo com sibilância audível pela ausculta pulmonar, hipertensão intracraniana (pressão intracraniana $>15 \mathrm{mmHg}$ ), fratura de arcos costais, pneumotórax não drenado, traqueostomizados, uso de suporte ventilatório com altos níveis de pressão positiva expiratória final (acima de 12 $\mathrm{cmH}_{2} \mathrm{O}$ ) e sistema de aspiração fechado.

Após a inclusão dos pacientes no estudo, a ordem de aplicação do protocolo de fisioterapia respiratória (PF) e do protocolo de aspiração traqueal isolada (PA) foi definida de forma aleatória. Envelopes pardos foram utilizados para o sorteio em blocos de 10. O intervalo entre a aplicação de cada protocolo foi estipulado em 24 horas, para não haver interferência do primeiro protocolo realizado. Os protocolos foram analisados isoladamente (intragrupo) e também analisados em conjunto (intergrupo), para comparar os dois protocolos entre si. As variáveis estudadas foram registradas em quatro momentos distintos: antes do protocolo, imediatamente após, 30 minutos e 120 minutos após a realização dos protocolos.

O PF consistiu em técnicas para remoção de secreções brônquicas: CTM (Compressão Torácica Manual) que consiste na compressão manual do tórax na fase expiratória na tentativa de deslocar as secreções de vias aéreas periféricas para as centrais; hiper-insuflação manual (HM) proporcionando um fluxo expiratório acelerado na fase expiratória; instilação de solução fisiológica com o objetivo de umidificar as secreções e aspiração traqueal (Tabela 1). O PA consistiu de aspiração traqueal somente. Ambas as aplicações foram realizadas pela mesma fisioterapeuta. O procedimento de aspiração traqueal realizado nos dois protocolos foi aplicado conforme as recomendações da American Association of Respiratory Care ${ }^{9}$.
Tabela 1 - Protocolo de Fisioterapia Respiratória

Compressão torácica manual por 10 minutos

Hiperinsuflação manual com $\mathrm{O}_{2}$ à $10 \mathrm{~L} / \mathrm{min}$

Instilação de 5 a $10 \mathrm{~mL}$ de solução fisiológica a 0,9\%

Aspiração traqueal

Hiperinsuflação manual com $\mathrm{O}_{2}$ à $10 \mathrm{~L} / \mathrm{min}$ (12 rpm) por 1 minuto

${ }^{*}$ American Association of Respiratory Care ${ }^{9}$

Os pacientes permaneceram em decúbito dorsal por, no mínimo, 20 minutos. Não foram realizados procedimentos de higiene corporal, radiografia torácica, administração de fármacos broncodilatadores e aspiração traqueal, por no mínimo, uma hora antes da aplicação dos protocolos. Para que não houvesse interferência nas variáveis mensuradas, os mesmos critérios foram utilizados até o último registro dos dados (duas horas após o protocolo).

Os dados demográficos, bem como medidas terapêuticas antes de cada protocolo, foram coletados. As variáveis estudadas consistiram em dados de mecânica pulmonar e cardiorrespiratórios: volume-minuto (VM), volume de ar corrente (VAC), complacência pulmonar dinâmica (Cdyn) e resistência do sistema respiratório (Rsr); freqüência cardíaca ( $F C)$, freqüência respiratória $(\mathrm{FR})$, pressão arterial sistólica (PAS), pressão arterial diastólica (PAD), pressão arterial média (PAM) e saturação periférica de oxigênio $\left(\mathrm{SpO}_{2}\right)$.

Os dados foram mensurados em quatro momentos distintos, para a avaliação do comportamento das variáveis com relação ao tempo de aplicação. O registro dos dados foi realizado antes e imediatamente após a aplicação de cada protocolo, 30 minutos e duas horas após.

As variáveis cardiorrespiratórias foram aferidas através do monitor (Siemens SC 7000 e 9000) contínuo conectado ao paciente, o qual registrou os sinais de FC, FR, $\mathrm{SpO}_{2}$, PAS, PAD e PAM. Os dados de ventilação-minuto e de mecânica pulmonar foram registrados através do ventilador mecânico (EVITA I e II, Dräger; Alemanha) que além de permitir o registro do VM e VAC, analisou e calculou os valores de Cdyn e Rsr.

Os dados com distribuição simétrica foram apresentados sob forma de média \pm DP ou porcentagem. A variável com distribuição assimétrica (tempo de VM) foi apresentada sob forma de mediana e percentis. Para a comparação das variáveis relacionadas ao tratamento medicamentoso e terapia ventilatória antes da aplicação dos protocolos foi utilizado o teste do Qui-quadrado de Mcnemar. Na comparação intragrupo foi utilizada a Análise de Variância (ANOVA) para medidas repetidas e como teste complementar utilizou-se o Least Significant Diffe- 
rence. Na comparação entre os grupos utilizou-se o teste $t$ de Student para as amostras pareadas. O nível estatístico significativo foi considerado quando $\mathrm{p}<0,05$.

\section{RESULTADOS}

No período de setembro a novembro de 2005, 12 pacientes completaram o estudo. As características iniciais dos pacientes no momento da inclusão no estudo estão demonstradas na tabela 2.

Tabela 2 - Características dos Pacientes Estudados

\begin{tabular}{lc}
\hline Número de Pacientes & 12 \\
\hline Idade (média \pm DP) & $75,6 \pm 14,5$ \\
Sexo & 10 \\
$\quad$ Masculino & 2 \\
$\quad$ Feminino & $21,6 \pm 6.8$ \\
APACHE II (média \pm DP) & $5(3-9,5)$ \\
VM dias (mediana) & \\
Causas da IRpA n (\%) & $5(41,7 \%)$ \\
Pulmonar & $4(33,3 \%)$ \\
Neurológica & $3(25 \%)$ \\
Cardíaca & \\
Comorbidades n (\%) & $6(50 \%)$ \\
Pulmonar & $1(8,3 \%)$ \\
Cardíaca & $2(16,7 \%)$ \\
Outra & \\
Terapia ventilatória n (\%) & \\
PCV & $4(33,3 \%)$ \\
PSV & $7(58,3 \%)$ \\
VCV & $1(8,3 \%)$ \\
PEEP (média \pm DP) & $6,5 \pm 1,2$ \\
FIO (média \pm DP) & $39,5 \pm 6,2$ \\
PIP (média \pm DP) & $14,2 \pm 4,2$ \\
Tratamento medicamentoso n (\%) & \\
Antibiótico & $10(8,3 \%)$ \\
Vasopressor & $4(33,3 \%)$ \\
Codação & $3(25 \%)$ \\
\hline
\end{tabular}

APACHE: Acute Physiology and Chronic Health Evaluation Score; VM: ventilação mecânica; PCV: ventilação pressão controlada; PSV: ventilação pressão de suporte; VCV: ventilação volume controlado; PEEP: pressão positiva expiratória final; $\mathrm{FIO}_{2}$ : fração inspirada de oxigênio; PIP: pressão inspiratória positiva.
As variáveis referentes à terapia medicamentosa e à ventilação mecânica utilizadas no momento antes da realização dos protocolos não apresentaram diferença significativa entre os grupos $(p=1,0)$. Nenhum dos pacientes do estudo fazia uso de bloqueador neuromuscular. A causa de ventilação mecânica foi predominantemente de doenças pulmonares ocorrendo em cinco pacientes $(41,7 \%)$, seguida de doenças neurológicas em quatro pacientes $(33,3 \%)$ e cardíacas em três pacientes (25\%).

Em quatro pacientes a doença mais comum foi à pneumonia, seguida de acidente vascular encefálico em dois pacientes e edema agudo pulmonar cardiogênico, também em dois pacientes.

Apenas três pacientes não apresentaram nenhuma doença associada. Das comorbidades encontradas a doença pulmonar obstrutiva crônica foi a mais comum, presente em cinco pacientes.

As variáveis cardíacas e respiratórias quando analisadas entre os grupos não apresentaram diferença estatística significativa em nenhum dos momentos avaliados (Tabela 3).

No grupo que realizou o $\mathrm{PA}$, a freqüência respiratória (FR) aumentou significativamente no momento imediatamente após a sua aplicação, comparada com o momento anterior à aplicação do protocolo (de $21 \pm 7$ rpm para $24 \pm 7 \mathrm{rpm} ; \mathrm{p}=0,02$ ), porém após os $30 \mathrm{~min}$, a variável retornou ao seu valor de base. A PAS no PA também teve aumento estatístico significativo após a aplicação do protocolo (de $110 \pm 37 \mathrm{mmHg}$ para $135 \pm$ $21 \mathrm{mmHg} ; \mathrm{p}=0,01$ ) retornando ao valor de base após 30 minutos.

Já no grupo que realizou o PF não foram encontradas alterações nos parâmetros cardiorrespiratórios em nenhum dos momentos do estudo. Entretanto, a $\mathrm{SpO}_{2}$ aumentou de forma significativa após o protocolo (de $97 \pm 2 \%$ para $99 \pm 2 \% ; p=0,01)$, mantendo-se acima do valor de base até os 120 minutos (Tabela 3).

Com relação à mecânica pulmonar, a Cdyn, VAC e VM

Tabela 3 - Variáveis Cardiorrespiratórias no Protocolo de Fisioterapia (PF) e Protocolo de Aspiração isolada (PA)

\begin{tabular}{lcccccccc}
\hline & \multicolumn{2}{c}{$\mathrm{FC}(\mathrm{bpm})$} & \multicolumn{2}{c}{$\mathrm{FR}(\mathrm{rpm})$} & \multicolumn{2}{c}{ PAM $(\mathrm{mmHg})$} & $\mathrm{SpO}(\%)$ \\
\hline & $\mathrm{PF}$ & $\mathrm{PA}$ & $\mathrm{PF}$ & $\mathrm{PA}$ & $\mathrm{PF}$ & $\mathrm{PA}$ & $\mathrm{PF}$ & $\mathrm{PA}$ \\
\hline Antes & $86 \pm 15$ & $87 \pm 19$ & $19 \pm 4$ & $21 \pm 7$ & $88 \pm 21$ & $83 \pm 17$ & $97 \pm 2$ & $98 \pm 1$ \\
Após & $89 \pm 14$ & $89 \pm 19$ & $20 \pm 5$ & $24 \pm 7^{*}$ & $96 \pm 18$ & $97 \pm 24$ & $99 \pm 2^{*}$ & $98 \pm 1$ \\
30 min & $90 \pm 16$ & $85 \pm 15$ & $19 \pm 3$ & $21 \pm 6$ & $90 \pm 19$ & $86 \pm 14$ & $98 \pm 2$ & $97 \pm 1$ \\
120 min & $86 \pm 15$ & $86 \pm 16$ & $19 \pm 3$ & $22 \pm 9$ & $87 \pm 15$ & $86 \pm 18$ & $98 \pm 2$ & $98 \pm 1$ \\
\hline
\end{tabular}

Valores expressos em média $\pm \mathrm{DP}$

* $p<0,05$ quando comparado com o momento antes da aplicação do protocolo

FC: freqüência cardíaca; FR: freqüência respiratória; PAM: pressão arterial média; $\mathrm{SpO}_{2}$ : saturação periférica de oxigênio 
ROSA, ROESE, SAVI E COL.

Tabela 4 - Variáveis de Mecânica Pulmonar no Protocolo de Fisioterapia (PF) e Protocolo de Aspiração Isolada (PA)

\begin{tabular}{lcccccc}
\hline & \multicolumn{2}{c}{ VAC $(\mathrm{L} / \mathrm{min})$} & \multicolumn{2}{c}{ VM $(\mathrm{L})$} & \multicolumn{2}{c}{$\mathrm{Cdyn}\left(\mathrm{mL} / \mathrm{H}_{2} \mathrm{O}\right)$} \\
\hline & PF & PA & PF & PA & PF \\
\hline Antes & $577 \pm 134$ & $606 \pm 172$ & $10,9 \pm 2,5$ & $11,5 \pm 2,2$ & $61,6 \pm 24,2$ & $61,7 \pm 25,5$ \\
Após & $554 \pm 194$ & $615 \pm 169$ & $11,7 \pm 3,6$ & $12,4 \pm 3,4$ & $65,6 \pm 25,5$ & $63,4 \pm 26,9$ \\
30 minutos & $616 \pm 130$ & $605 \pm 166$ & $11,0 \pm 3,3$ & $11,6 \pm 3,2$ & $65,5 \pm 26,9$ & $62,6 \pm 25,4$ \\
120 minutos & $618 \pm 151$ & $599 \pm 180$ & $11,4 \pm 2,9$ & $11,5 \pm 2,7$ & $65,6 \pm 25$ & $60,6 \pm 25,2$ \\
\hline
\end{tabular}

Valores expressos em Média \pm DP

VAC: volume de ar corrente; VM: volume-minuto; Cdyn: complacência dinâmica

não alteraram significativamente em nenhum dos momentos avaliados, tanto na análise intragrupo como na análise intergrupo (Tabela 4). Em contrapartida, a Rsr diminuiu no PF significativamente nos momentos imediatamente após (de 10,4 $\pm 3 \mathrm{cmH}_{2} \mathrm{O} / \mathrm{L} /$ seg para $8,9 \pm$ $2 \mathrm{cmH}_{2} \mathrm{O} / \mathrm{L} / \mathrm{seg} ; \mathrm{p}<0,02$ ), 30 minutos após (de 10,4 \pm $3 \mathrm{cmH}_{2} \mathrm{O} / \mathrm{L} / \mathrm{seg}$ para $\left.9 \pm 2 \mathrm{cmH}_{2} \mathrm{O} / \mathrm{L} / \mathrm{seg} ; \mathrm{p}<0,01\right) \mathrm{e}$ 120 minutos após (de 10,4 $\pm 3 \mathrm{cmH}_{2} \mathrm{O} / \mathrm{L} / \mathrm{seg}$ para $9 \pm 2$ $\left.\mathrm{cmH}_{2} \mathrm{O} / \mathrm{L} / \mathrm{seg} ; \mathrm{p}<0,03\right)$. Esses valores também foram menores quando comparados com o PA nos momentos $30 \mathrm{~min}\left(9 \pm 2 \mathrm{cmH}_{2} \mathrm{O} / \mathrm{L} / \mathrm{seg}\right.$ versus $10,2 \pm 2 \mathrm{cmH}_{2} \mathrm{O} / \mathrm{L}$ seg, $\mathrm{p}<0,04)$ e $120 \mathrm{~min}\left(9 \pm 2 \mathrm{cmH}_{2} \mathrm{O} / \mathrm{L} /\right.$ seg versus 10,4 $\left.\pm 3 \mathrm{cmH}_{2} \mathrm{O} / \mathrm{L} / \mathrm{seg}, \mathrm{p}<0,04\right)$. Após a aplicação do PA, não houve alterações significativas na Rsr (Figura 1).

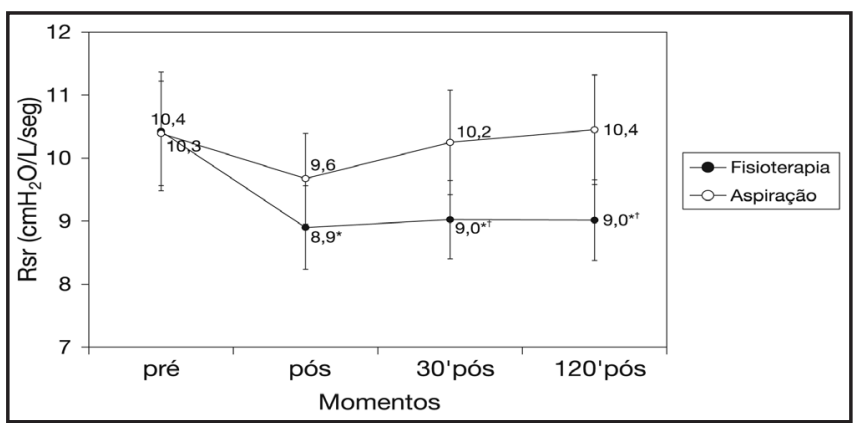

Figura 1 - Resistência do Sistema Respiratório (Rsr)

${ }^{*} p<0,02 ; p<0,01 ; p<0,03$, respectivamente na análise intragrupo com relação ao momento antes da aplicação do protocolo.

† $p<0,04$ na análise entre os grupos comparados no mesmo momento.

\section{DISCUSSÃO}

As técnicas de desobstrução brônquica são freqüentemente utilizadas em pacientes em VMl a fim de prevenir o acúmulo de secreções respiratórias e diminuir o risco de pneumonia associada ao ventilador ${ }^{3,10}$. Muitas técnicas desobstrutivas são utilizadas para este fim, porém a maioria dos estudos avalia técnicas isoladas com protocolos variados.

Este estudo avaliou os efeitos de um protocolo de higiene brônquica em curto prazo, comparando com protocolo controle, em que foi realizada apenas a as- piração do tubo traqueal. As alterações cardiorrespiratórias foram observadas apenas no PA, imediatamente após a sua aplicação, em que houve aumento não sustentado da FR e PAS. A aplicação de pressão negativa durante a aspiração pode colapsar algumas zonas aéreas, deteriorando a oxigenação e aumentando o trabalho ventilatório ${ }^{6}$. Esse aumento não foi observado no PF, possivelmente por ter sido aplicado a HM após o procedimento de aspiração, com o objetivo de ventilar e insuflar os pulmões com fluxo de oxigênio de $10 \mathrm{~L} / \mathrm{min}$. Estudos demonstraram que o uso da HM ocasiona abertura de vias aéreas colapsadas e melhora da oxigenação sem repercutir em parâmetros cardíaco e respiratório ${ }^{11,12}$. Em concordância com este estudo, Paratz e col. não detectaram alterações nos parâmetros cardiorrespiratórios após uso da HM isolada em pacientes com lesão pulmonar aguda ${ }^{13}$. Hodgson e col. também não identificaram essas alterações comparando aspiração isolada com HM combinada com aspiração em 18 pacientes com doenças variadas $^{7}$. Mackenzie e Shin ${ }^{14}$ avaliaram as repercussões das técnicas manuais aplicadas sobre o tórax em 19 pacientes pós-traumatizados em VMI, não observando alterações das funções cardíaca e respiratória.

$\mathrm{A} \mathrm{SpO}_{2}$ aumentou significativamente após o PF não apresentando o mesmo comportamento quando realizado o PA. A remoção de secreções eficaz e a ventilação proporcionada pela HM após a aspiração, podem ter gerado um efeito positivo após a aplicação do PF. Alguns estudos têm demonstrado os efeitos da HM na resolução de atelectasias e remoção de secreções respiratórias, agindo diretamente na melhora da saturação de oxigênio ${ }^{4,7,11}$.

No presente estudo, os valores de Cdyn, VAC e VM não alteraram de forma significativa em nenhum dos protocolos realizados. Unoki e col. realizaram estudo cruzado em 31 pacientes em VMI com doenças variadas $^{8}$. Eles compararam um protocolo de CTM por três minutos com aspiração isolada e não encontraram diferença significativa nos valores de Cdyn entre os grupos. Em contrapartida, o aumento da Cdyn foi obser- 
vado em estudos com grupos de pacientes com doenças específicas (lesão pulmonar aguda e pneumonia) e gerais, mantendo-se superior até duas horas após a intervenção ${ }^{7,13,15}$. Neste estudo o número reduzido da amostra e a variedade de doenças dos pacientes talvez justifiquem este resultado, visto que a maioria dos estudos detectou aumento da Cdyn após a aplicação de técnicas desobstrutivas.

A Rsr diminuiu de forma estatisticamente significativa apenas no PF. Comparando com o momento pré-protocolo, todos os momentos posteriores avaliados foram significativamente menores. Comparando com o PA, a Rsr foi menor nos 30 e 120 min após a aplicação do protocolo. Esse dado sugere que o FR promove higiene brônquica eficaz, através do deslocamento das secreções das vias aéreas de menor calibre para as mais centrais, fazendo com que maior volume de secreção seja removido com a aspiração traqueal. Choi e col., em estudo prospectivo cruzado, avaliaram o efeito da HM na mecânica pulmonar em 15 pacientes, com pneumonia associada ao ventilador comparando com aspiração isolada ${ }^{15}$. A Rsr diminuiu significativamente após o protocolo de HM, comparado com o controle e manteve-se menor até os 30 minutos após o protocolo.

Não existem estudos relacionados à diminuição da Rsr após FR no que diz respeito ao ajuste do ventilador mecânico, uso de fármacos broncodilatadores, trabaIho respiratório, morbidade e desfecho clínico desses pacientes. Novos estudos devem ser realizados com o objetivo de avaliar os benefícios da diminuição da Rsr na evolução clínica dos pacientes submetidos à VMI.

Esta pesquisa apresenta algumas limitações, as quais impossibilitam a generalização dos resultados. O aumento da amostra se faz necessário para avaliação das variáveis que não alteraram significativamente, além disso, a variedade das doenças de base pode ter interferido nos resultados, principalmente no que diz respeito à Cdyn.

Apesar de alguns estudos demonstrarem efeitos positivos em relação às técnicas de higiene brônquica, é imensa a variedade de protocolos no que tange a técnicas e tempo de aplicação ${ }^{7,10,11,13}$. Estudos sobre esse tema são necessários para enriquecer a discussão sobre os efeitos da fisioterapia respiratória nesses pacientes e para haver consenso com relação às técnicas que podem ser utilizadas e quais os benefícios esperados após a sua aplicação. Protocolos que reproduzam a prática clínica, com aplicação de técnicas combinadas, em grupos de pacientes com doenças específicas podem auxiliar no melhor manuseio fisioterapêutico nos pacientes críticos em VMI.

Neste estudo, a Rsr comportou-se de forma favorável até duas horas após o protocolo de fisioterapia respiratória, não ocorrendo no grupo que realizou apenas a aspiração traqueal isolada. Não foi identificada alteração na complacência pulmonar.

\section{AGRADECIMENTOS}

Agradecimento especial a todos os fisioterapeutas do CTI Adulto do Hospital Moinhos de Vento que contribuíram para que esta pesquisa tenha sido realizada: Ricardo Wickert, Luis Guilherme Borges, Kamile Borba Pinto, Fernanda Gehn, Augusto Savi, Fernanda Callefe, Priscila Pereira e Maurem Porto.

\section{REFERÊNCIAS}

01. Gayan-Ramirez G, Decramer M - Effects of mechanical ventilation on diaphragm function and biology. Eur Respir J, 2002;20:1579-1586.

02. Tobin MJ - Mechanical ventilation. N Eng J Med, 1994;330:1056-1061.

03. Judson MA, Sahn SA - Mobilization of secretions in ICU patients. Respir Care, 1994;39:213-226.

04. Stiller K - Physiotherapy in intensive care: towards and evidence-based practice. Chest, 2000;118:1801-1813.

05. Ciesla N - Chest physical therapy for patients in the intensive care unit. Phys Ther, 1996;76:609-625.

06. Selsby D, Jones JG - Some physiological and clinical aspects of chest physiotherapy. Br J Anaesth, 1990;64:621-631.

07. Hodgson $\mathrm{C}$, Denehy L, Ntoumenopoulos $\mathrm{G}$ et al - An investigation of the early effects of manual lung hyperinflation in critically ill patients. Anaesth Intensive Care, 2000;28:255-261.

08. Unoki T, Kawasaki Y, Mizutani T et al - Effects of expiratory rib-cage compression on oxygenation, ventilation, and airway secretion removal in patients receiving mechanical ventilation. Respir Care, 2005;50:14301437.

09. AARC Clinical Practice Guideline. Endotraqueal suctioning of mechanically ventilated adults and children with artificial airways. Respir Care 1993;38:500-5004.

10. Ntoumenopoulos G, Presneill JJ, McElholum M et al - Chest physiotherapy for the prevention of ventilator-associated pneumonia. Intensive Care Med, 2002;28:850-856.

11. Maa $\mathrm{SH}$, Hung TJ, Hsu KH et al - Manual hyperinflation improves alveolar recruitment in difficult-to-wean patients. Chest, 2005;128:27142721.

12. Denehy $L-$ The use of manual hyperinflation in airway clearance. Eur Respir J, 1999;14:958-965.

13. Paratz J, Lipman J, McAuliffe M - Effect of manual hyperinflation on hemodynamics, gas exchange, and respiratory mechanics in ventilated patients. J Intensive Care Med, 2002;17:317-324.

14. Mackenzie CF, Shin B - Cardiorespiratory function before and after chest physiotherapy in mechanically ventilated patients with post-traumatic respiratory failure. Crit Care Med, 1985;13:483-486.

15. Choi JS, Jones AY - Effects of manual hyperinflation and suctioning in respiratory mechanics in mechanically ventilated patients with ventilatorassociated pneumonia. Aust J Physiother, 2005;51:25-30. 